\title{
DIGITALIZATION IN CONSTRUCTION: BLOCKCHAIN APPLICABILITY IN THE INDUSTRY
}

\author{
GIULIA PATTINI $^{1}$, ELENA SEGHEZZI ${ }^{1}$, and GIUSEPPE MARTINO DI GIUDA ${ }^{2}$ \\ ${ }^{I}$ Dept of ABC, Politecnico di Milano, Milano, Italy \\ ${ }^{2}$ Dept of Management, Università degli Studi di Torino, Torino, Italy
}

\begin{abstract}
Blockchain, as a Distributed Ledger Technology (DLT), could disrupt the traditional hierarchy way in which information is shared and processes are developed in the construction industry. The fragmentation of people, processes and contracts has been considered as the main responsible for the sector negative trend. The information asymmetry characterizing the dynamics of the processes denies both the information traceability and the disciplines interoperability. The recent digital transformation boosted by Building Information Modeling (BIM) has promised a more transparent and collaborative environment. However, its adoption has gradually shown some trust issues in the information flow, discouraging participants from collaborating to achieve project goals. For these reasons, the research explores Blockchain in the construction industry, highlighting its potentials in supporting transparent, reliable and distributed information sharing. In particular, the research intends to combine the features of the digitalization, namely BIM and Blockchain, in the industry. To accomplish this, the first goal consists of an overall literature review on the current level of the Blockchain investigation in the built environment. Secondly, based on that analysis, two proposals for potential application of Blockchain in AECO sector are presented. This preliminary exploration intends to endorse Blockchain as a suitable system for governing the trust issues, supporting an efficient contract execution and a better sharing of all project information.
\end{abstract}

Keywords: BIM, Smart Contracts, Process automation, Trust, Collaborative environment, Information management.

\section{DIGITAL TRANSFORMATION IN CONSTRUCTION}

The current AECO sector digitalization is driven by the combination of Building Information Modeling, Internet of Things (IoT) and cloud services. These innovations ensure interconnected buildings that can be created, monitored and managed in real-time. Among these digital changes the Distributed Ledger Technologies, in particular, Blockchain, are in their outset. For these reasons, the research investigates Blockchain as a breakthrough technology for the industry.

The recent poor performance of the sector has encouraged the stakeholders to change their traditional procedures to achieve more suitable results (Farmer 2016). Some professionals have indeed progressively proposed transformations of the methods and implementations of the technologies aimed at overcoming the issues and redefining the traditional industry schemes. In particular, the adoption of new digital approaches to the construction process is the key to ensure compliance with the requirements of the client and all stakeholders. The first impetus for change lies in the digitization of information exchanges, that entails the reorganization of process relationships and approaches (Mosey et al. 2016). Namely, BIM has been proposed as a method 
to support the collaborative development of the project. Based on a shared platform, containing all the documents and information, BIM promotes information sharing and cooperation among the parties (Antwi-Afari et al. 2018). Digital environment significantly influences relationships by supporting the whole project planning and execution and visualization of its evolution.

This innovative methodology permits parties to manage a high amount of data and to constantly control the project development. Thus, it is possible to monitor the gap between the expected and actual quality, reducing the occurrence of delays and disputes (Tezel et al. 2019, Stougiannos and Magneron 2018).

The interdisciplinary nature of the sector brings multiple actors to interact and enrich, even simultaneously, the project with different digital information. Unfortunately, these activities have progressively highlighted cybersecurity and data privacy issues. In particular, the uncertainties regarding the reliability, integrity and completeness of the information shared in the digital environment are the main ones (Turk and Klinc 2017). In the long term, the presence of such problems is likely to lead back to traditional process disputes. The lack of trust in information and relationships discourages participants from collaborating, favouring the pursuit of opportunistic behaviour. Such a situation shatters the innovative digital transformation goals.

For these motivations, among the innovations that are slowly gaining ground, the integration of Blockchain technology is proposed. Blockchain structure can facilitate security, accountability and traceability of data. An immutable digital ledger provides control and tracking of the project at every stage (Kassem et al. 2018). Summarizing, given the introduction above, this research is structured in the fulfillment of two main purposes (Figure 1). The first goal, discussed in section 2 , provides an overview of the potential application areas of Blockchain in the construction industry, detected in the existing literature. The second goal, discussed in section 3, explores the applicability of Blockchain in the industry, suggesting two potential proposals.

The proposed research is at an early and exploratory stage. The main intent is to provide an approach for investigating the technology in the industry. The issues illustrated stand as an incentive for future in-depth analysis on the integration of BIM and Blockchain, to support both better information sharing and efficient contract execution.

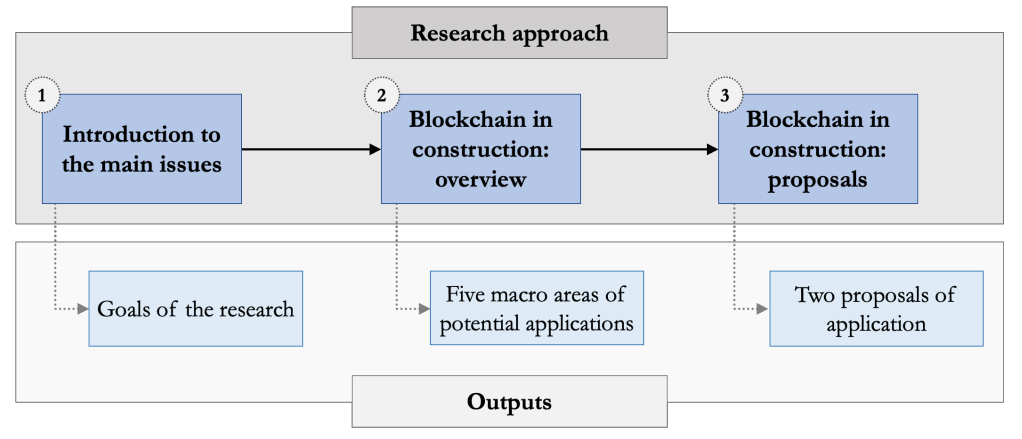

Figure 1. Scheme of the presented research approach.

\section{A THEORETICAL OVERVIEW OF BLOCKCHAIN IN CONSTRUCTION}

With its ability to revolutionize the dynamics of multiple sectors and redefine business processes, Blockchain has been regarded as the primary technology of the current digital transformation. Blockchain consists of a virtual "chain of blocks" that develops internally to a distributed database on which are recorded series of encrypted operations in time sequence. Each block represents a link in the chain, initially completed and validated with the information related to the 
operations received and then closed and marked with its identification code, thus becoming immutable. Each new block is located at the top of the chain, creating a database of all transactions in chronological order. Blockchain can be defined as a system that provides the acquisition of a certain data in computer format, making it true, monitorable, immutable without the need for a third-party authority. The information entered and recorded on Blockchain is therefore verified and validated by the entire network, through the consensus mechanism.

Again, the programmable nature of the Blockchain enables the correct use of Smart Contracts. These are computerized transaction protocols that execute the coded terms of a contract automatically. These programmed codes highlight the revolution of the traditional contractual system where the parties draft the essential terms of the agreement using a descriptive language. Indeed, Blockchain promotes the representation of clauses in the form of structured data capable of self-executing through computer protocols.

Although the adoption of the technology has originally mainly affected the financial and governance sectors, recently also the construction sector has shown an increasing interest (ARUP 2019). The features of the technology could help to overcome and contain the main conflicts of the industry. Its distributed nature could support the creation of a shared, collaborative and transparent environment among all the participants. Its integration into the process could ensure the workflow optimization, from the design phases to the building maintenance (Hughes 2017). As anticipated in the introduction, in this section the Blockchain overview in the construction sector is based on the analysis of the existing scientific literature. Although, to date, there are no actual adoptions, the study of the publications has outlined some common conceptual applications, that can be grouped into five areas of use, as described below (Erri Pradeep et al. 2019, Hunhevicz and Hall 2020, Scott et al. 2020, Wang et al. 2017).

\subsection{Digital Document Management}

Due to increased complexity of project requirements and specifications, each step of the process requires the creation and exchange of a large amount of information. Blockchain integration improves the repository environment by bringing trust and integrity to the data shared. Thanks to Blockchain, documents are stored on a common distributed platform among all participants, which ensures the notarization of any data created, modified and updated as process progresses.

\subsection{Contract Management}

In the construction contractual environment, the possibility of making automatic certain processes, that are traditionally entrusted to interaction and pursued based on multiple professionals' decisions, is a valid area of experimentation. Smart Contracts, as codified protocols implemented through Blockchain, can be integrated or combined with the drafting of certain clauses of traditional agreements. The codified clauses are thus programmed ex-ante and executed autonomously when a certain event occurs.

\subsection{Building Information Modeling}

The BIM model is a centralized source of lots of information, continuously updated, modified and validated. However, it is not always possible to check or assess the entities responsible for these activities, discouraging the parties to cooperate to achieve the common contractual goals. The management of the BIM model using Blockchain technology makes it possible to record any activity and therefore to control "who, what and when" at all times. In this way, a single source of truthful information and a legal basis for any litigation is created. 


\subsection{Supply Chain Management}

Due to the high number of operators, the supply chain often pursues an unstable and fragmented trend, with consequent disregard of time, cost and work quality. To prosecute an efficient and sustainable development of the process, the data accuracy and consistency in the supply chain are essential. The adoption of Blockchain supports truthful monitoring of what is happening, making the information known to all those involved.

\subsection{Real Estate Management}

As a distributed registry, it is possible to notarize on Blockchain the structures of the real estate assets. In this way, public administrations and individuals can control ownership of the property and manage the registration, purchase and transfer of the property in a transparent manner.

\section{BLOCKCHAIN IN CONSTRUCTION: TWO PROPOSALS}

The overview of Blockchain in construction has permitted the identification of five theoretical areas of application. The progressive use of the BIM method has supported the transformation of both the approaches and the organizations. This method, encouraging the data digitization, requires from the very first moment the planning and structuring of the entire information flow. As a result, this new digital environment supports the spontaneous integration of Blockchain technology and its Smart Contracts protocols. As mentioned in the introduction, this section suggests two proposals for the application of the technology, highlighting how it can influence the overall contract execution and, consequently, the performance trend. The two proposals are focused on the achievement of a BIM process based on Blockchain, supporting the decisionmaking processes, and on the Smart Contracts adoption, facilitating the cash flow management.

\subsection{BIM Process Based on Blockchain}

In line with the overview illustrated, the first proposal concerns the development of a Blockchainbased BIM process. BIM is currently the main promoter of the creation, sharing and management of project information. Blockchain could instead be considered as a technology that ensures transparency, trust and certainty of the information exchanged (McNamara 2020). As a Distributed Ledger Technology, Blockchain could make the BIM model achieve a higher level of transparency, verifiability and effectiveness. Their integration ensures that every change in the model is accurately recorded. In this way, an increase in reliability, traceability and ownership of information is achieved, improving collaboration and trust among the parties involved. The pairing of BIM and Blockchain ensures the development of a digital environment that is no more centralized but distributed. In there, information is collected, maintained, updated and stored in a reliable and unchangeable way (Nawari and Ravindran 2019). Distributed network ensures the trust of the information, giving access and control at any time. Simplicity of information consultation supports the decision-making process, from the design phase to the maintenance of the building, carrying forward a sustainable development of the project. This environment makes it possible to constantly monitor the progress of the work by those involved; each project participant is encouraged to engage in the execution of their tasks in accordance with the common contractual objectives (Koutsogiannis and Berntsen 2017).

\subsection{Smart Contracts Breakthrough}

If the advent of BIM supported a data-driven approach influencing the use of information, holding up the shift from unstructured information to structured information. The mere advent of 
Blockchain will lead to the transformation of contractual clauses, from expressions in descriptive language to expressions in computational language, abandoning the opportunistic behavior due to a free interpretation of the traditional contract clauses.

Still in line with what has been investigated in the state of the art, the second proposal concerns the use of Smart Contracts to support the traditional execution of the contract. Traditional contracts that are formed through a long period of negotiation between the parties, have elaborate drafting stages and require the contribution of lawyers to establish the obligations of the contracting party. The "manual" nature of the traditional process is rich in inconsistent and ambiguous drafting practices that are at the root of most disputes in the industry.

For these reasons, Smart Contracts are a key element for the efficient development of the clauses. Smart Contracts, thanks to their programmable and automatic nature, can eliminate the need for a trusted third party, administering the contract in a truly autonomous way, and integrating Building Information Modeling and the Internet of Things, to "inform" Smart Contracts of the progress of the work and the actual building performance ( $\mathrm{Li}$ et al. 2019). Besides, the adoption of Smart Contracts, based on the distributed Blockchain environment, fits with the nature of collaborative agreements created to regulate and support the use of BIM.

\section{CONCLUSION AND FURTHER DEVELOPMENTS}

The revolution of information organization, production and management, driven by the adoption of BIM, requires the replacement of traditional competitive strategies with more collaborative ones. So, the intention to implement Blockchain technology in a BIM process is an opportunity to create a streamlined construction process, based on a trusted and collaborative approach.

As stated in the first proposal, integrating the Blockchain platform into the BIM process could improve communication among the parties, ensuring better project planning and monitoring, better leadership and efficient traceability of the entire supply chain. Furthermore, as stated in the second proposal, the possibility of using Smart Contracts in support of the traditional contract framework would guarantee the respect of contract clauses and timely payment. Thanks to the discussion carried out, main areas of applicability of Blockchain cloud be summarized in:

- Information management: the distributed nature permits the information to be created and shared simultaneously and each document (drawings, models, certifications, datasheets, work schedule, etc.) to be archived reliably and immutably. Every data becomes transparent and notarized on the distributed platform.

- Cash flow management: project milestones can be notarized on the platform and payment can be scheduled for each of them. The use Smart Contracts permits the automatic release of payments when the planned condition is met, saving time and money that are usually due by the need of a third party or the resolution of any disputes.

Despite the benefits deduced from the theoretical applications presented, the revolutionary nature of the technology does not suggest that traditional parties are willing to easily rely on this new system. The distributed environment leads the stakeholders to question both the sharing of business information and the actual success of implementing the technology in their activities. The functioning of the technology requires the presence of personnel experienced in coding and the programmability of Smart Contracts does not leave room for contractual changes. In this sense, the unpredictable nature of the process in the long term and the incompatibility between Blockchain applications and existing regulatory schemes are major obstacles to the technology deployment. In the same way as with BIM, it is only through the progressive enactment of common regulations and will that it is possible to encourage industry professionals to adopt Blockchain, to make it an ordinary technology as is already the case in other industrial sectors 
(Osservatorio Blockchain \& Distributed Ledger 2020). Therefore, to be able to propitiate the adoption and integration of these technologies, a change of mentality and specific training is necessary, as well as challenging.

The advancement of Blockchain in the digital transformation of the industry would bring sufficient change to modify, even if only partially, relational and methodological practices. This would enable the industry to better manage information and resources, thereby reducing legal costs, project duration and disputes. For these reasons, based on what discussed in this introductory research, it is necessary to explore the presented issues in more detail and depth. Future researches will show the correct ways of integrating the technology into the sector and the most appropriate phases for its adoption.

\section{References}

Antwi-Afari, M. F., Li, H., Pärn, E. A., and Edwards, D. J., Critical success factors for implementing building information modelling (BIM): A longitudinal review, Automation in Construction, vol. 9, 100-110, July, 2018.

ARUP, Blockchain and the Built Environment, 2019.

Erri Pradeep, A. S., Yiu, T. W., and Amor, R., Leveraging Blockchain Technology in a BIM Workflow: A Literature Review, 371-80, January, 2019.

Farmer, M., The Farmer Review of the UK Construction Labour Model, Modernise or die: The Framer Review of the UK construction labour market. Retrieved from, 2016.

Hughes, D., The Impact of Blockchain Technology on the Construction Industry, 2017.

Hunhevicz, J. J., and Hall, D. M., Do you need a blockchain in construction? Use case categories and decision framework for DLT design options, Advanced Engineering Informatics, 45, August, 2020.

Kassem, M., Li, J., and Greenwood, D., Blockchain in the built environment: analysing current applications and developing an emergent framework, 59-66, 2018.

Koutsogiannis, A., and Berntsen, N., Blockchain and construction: the how, why and when, Chartered Institute of Building, 2017.

Li, J., Kassem, M., Ciribini, A. L. C., and Bolpagni, M., A Proposed Approach Integrating DLT, BIM, IoT and Smart Contracts: Demonstration Using a Simulated Installation Task, 275-82, 2019.

McNamara, A., Automating the Chaos: Intelligent Construction Contracts, In IntechOpen, 20, 2020.

Mosey, D., Bahram, D., Howard, C., Dartnell, R., Hallam, C., Howard, C., Maqbool, A., Murray, K., Rawlinson, S., and Winfield, M., Enabling BIM through procurement and contracts, Society of Construction Law Papers D, 67, 2016.

Nawari, N. O., and Ravindran, S., Blockchain Technologies in BIM Workflow Environment, 593-602, 2019.

Osservatorio Blockchain \& Distributed Ledger, Blockchain and Distributed Ledger: unlockcing the potential of the Internet of Value, 2020.

Scott, D. J., Broyd, T., and Ma, L., Archival Study of Blockchain Applications in the Construction Industry From Literature Published in 2019 and 2020, Proceedings of the ARCOM Doctoral Workshop, 2020.

Stougiannos, L., and Magneron, A., BIM, Blockchain and the Smart Construction Contract - Lexology, Lexology, 3-6, 2018.

Tezel, A., Papadonikolaki, E., and Yitmen, I., Preparing Construction Supply Chains for Blockchain: An Exploratory Analysis, CIB World Building Congress, June 17-21, 2019.

Turk, Ž., and Klinc, R., Potentials of Blockchain Technology for Construction Management, Procedia Engineering, 196, 638-45, June, 2017.

Wang, J., Wu, P., Wang, X., and Shou, W., The outlook of blockchain technology for construction engineering management, Frontiers of Engineering Management, 4(1), 67, 2017. 